\title{
Neural Networks Design: Rough Set Approach to Continuous Data*
}

\author{
Nguyen Hung Son, Marcin S. Szczuka, Dominik Ślęzak \\ Institute of Mathematics, Warsaw University \\ Banacha 2, 02-097 Warsaw, Poland \\ email: szczuka@mimuw.edu.pl, \{son,slezak\}@alfa.mimuw.edu.pl
}

\begin{abstract}
The construction of neural network based on decision rules generated by rough set methods is given. Analogies between neural network semantics and rough set approach to continuous data are pointed out. General framework states the initial point for such applications like, e.g., tuning decision rules by neural network learning process.
\end{abstract}

\section{Introduction}

The task of dealing with real-valued datasets seems to be one of key issues in decision support systems. Many approaches have been developed so far. One of them is to scale attributes and apply rough set techniques over discrete-valued decision tables obtained. If decision rules with scaled outputs are of satisfactory quality for the user, then tools developed in [5],[6],[7] seem to be appropriate. However, in many applications, like e.g. control processes, there is a strong need to go back to real-valued shape of decision, after performing rough set analysis over scaled data. Then, one can say that rough set decision rules correspond to so called linguistic rules in fuzzy reasoning ([4],[9]). The main challenge while inferring about real-valued decision is to find state equations for fuzzy variables, corresponding to conditions in rough set decision rules.

It is worth mentioning that neural networks themselves are widely used as decision support algorithms. However, although such an approach is very powerful, it has some restrictions. First, one may have difficulties with finding proper layout of the network (e.g. number of neurons or layers). Second, network itself does not provide us with clear interpretation of knowledge it contains ([9]). Fortunately, rough set methods ([7],[8]) can help to construct initial network in terms of such parameters like the numbers of scaling conditions, minimal decision rules and decision classes in discrete case. Then, the threshold functions in neurons correspond to fuzzyfication of rough set rules and the weights - to equations of scaling conditions and the degree of approximation for particular decision patterns.

* This paper was supported by the State Committee for Scientific Research grant, KBN 8T11C01011. 


\section{Rough sets and discretization}

\subsection{Information systems}

Information system is a pair $\mathbf{A}=(U, A)$ where $U$ is a non-empty, finite set called the universe and $A$ is a non-empty, finite set of attributes, i.e. $a: U \rightarrow V_{a}$ for $a \in A$, where $V_{a}$ is called the value set of attribute $a$. Elements of $U$ are called objects.

Every information system $\mathbf{A}=(U, A)$ and a non-empty set $B \subseteq A$ define a $B$-information function by $\operatorname{In} f_{B}(u)=\left(a_{i}(u): a_{i} \in B\right)$ for $u \in U$ and some linear order $A=\left\{a_{1}, \ldots, a_{n}\right\}$. The set $\left\{I n f_{B}(u): u \in U\right\}$ is called the $B$-information set and it is denoted by $V_{B}$.

In case of real-valued attributes, where for each $i \leq n a_{i}: U \rightarrow \mathbf{R}$ is a real function from universe $U$, its elements can be characterized as points:

$$
P_{u}=\left(a_{1}(u), a_{2}(u), \ldots, a_{n}(u)\right)
$$

in $\boldsymbol{n}$-dimensional affine space $\mathbf{R}^{n}$. The validity of such a representation is based on assumption that for $A$-indiscernibility relation $I N D(A)$ (which can be associated with any $B \subseteq A$ as well) defined by

$$
I N D(B)=\left\{\left(u, u^{\prime}\right) \in U \times U: \operatorname{In} f_{B}(u)=\operatorname{In} f_{B}\left(u^{\prime}\right)\right\}
$$

all equivalence classes are singletons corresponding to particular objects in $U$.

\subsection{Optimal scaling in decision tables}

A decision table is any information system of the form $\mathbf{A}=(U, A \cup\{d\})$, where $d \notin A$ is a distinguished attribute called decision. The elements of $A$ are called conditions.

We assume that the set $V_{d}$ of values of the decision $d$ is equal to $\left\{v_{1}, \ldots, v_{n_{d}}\right\}$ for some positive integer $n_{d}$ called the range of $d$.

The decision $d$ determines the partition $\left\{C_{1}, \ldots, C_{n_{d}}\right\}$ of the universe $U$, where $C_{l}=\left\{u \in U: d(u)=v_{l}\right\}$ for $1 \leq l \leq n_{d}$. The set $C_{l}$ is called the $l$-th decision class of $\mathbf{A}$.

For a decision table with real-valued conditions, according to the assumption about discernibility of objects with respect to $I N D(A)$, any of them belongs to one of the decision classes $C_{1}, C_{2}, \ldots, C_{n_{d}}$ where

$$
C_{l}=\left\{u \in U: d(u)=v_{l}\right\}
$$

The main task is how to approximate these classes by possibly small and regular family of subsets $\tau_{k} \subseteq \mathbf{R}^{n}$, where any $\tau_{k}$ points out at some decision value $v_{l(k)}$ , e.g., in terms of its high frequency of occurrence for objects in $\tau_{k}$.

In [6] searching for such decision rules were performed by defining hyperplanes over $\mathbf{R}^{n}$. Any hyperplane

$$
H=\left\{\left(x_{1}, x_{2}, \ldots, x_{n}\right) \in \mathbf{R}^{n}: \alpha_{0}+\alpha_{1} x_{1}+\cdots+\alpha_{n} x_{n}=0\right\}
$$


(where $\alpha_{0}, \alpha_{1}, \alpha_{2}, \ldots, \alpha_{n} \in \mathbf{R}$ ) splits $C_{l}$ onto two subclasses defined by:

$$
C_{l}^{U, H}=\left\{u \in C_{l}: H(u) \geq 0\right\} \text { and } C_{l}^{L, H}=\left\{u \in C_{l}: H(u)<0\right\}
$$

where, for a given hyperplane, function $H: U \rightarrow \mathbf{R}$ is defined by $H(u)=$ $H\left(I n f_{A}(u)\right)$.

Let us introduce an examplar measure estimating the quality of hyperplanes with respect to the decision classes $C_{1}, C_{2}, \ldots, C_{n_{d}}$. Consider function

$$
\operatorname{award}(H)=\sum_{l 1 \neq l 2} \operatorname{card}\left(C_{l 1}^{U, H}\right) \cdot \operatorname{card}\left(C_{l 2}^{L, H}\right)
$$

If $\operatorname{award}(H)>\operatorname{award}\left(H^{\prime}\right)$ for some hyperplanes $H, H^{\prime}$, then the number of pairs of objects from different decision classes discerned by $H$ is greater than the corresponding number for $H^{\prime}$. Thus, this is $H$ which should be considered while building decision rules. If $H$ does not discern enough number of pairs, then one can search for next hyperplanes, until obtaining satisfactory degree of decision classes' approximation. By $n_{h}$ we denote the number of hyperplanes found by such algorithm.

The number of decision rules, equal to $2^{n_{h}}$ due to all possible combinations of position of objects with respect to $n_{h}$ hyperplanes, can be reduced to the number $n_{r} \leq 2^{n_{h}}$ of minimal decision rules of the form $\tau_{k} \Rightarrow d=v_{l(k)}$, where no component $\tau_{k j}$ corresponding to hyperplane $H_{j}$ can be rejected without decrease of given degree of approximation.

\section{Hyperplane-based network}

\subsection{Initial construction}

Once the hyperplanes and decision rules are constructed for given A, we may put them into the neural network. It is worth noticing that, given decision table $\mathbf{A}=(U, A \cup\{d\})$ and the set of $n_{h}$ hyperplanes inducing $n_{r}$ decision rules, one can construct four-layer neural network with $n+1$ inputs, $n_{h}$ and $n_{r}$ neurons in hidden layers respectively, and with $n_{d}$ outputs, such that it recognizes objects in $U$ just like in case of corresponding hyperplane decision tree.

Such a network has $n$ inputs corresponding to conditional attributes and one additional constant input called bias. Every input neuron sends its signal to all neurons in hidden layer. For each hyperplane from $H$ we construct one neuron in the hidden layer. This neuron has weights equal to coefficients describing corresponding hyperplane.

For all neurons in the first hidden layer the threshold functions have the form

$$
h_{j}(x)=\left\{\begin{array}{c}
1 \text { for } x \geq 0 \\
-1 \text { for } x<0
\end{array}\right.
$$

This is also the case for thresholds in the second layer, which are given as

$$
r_{k}(x)=\left\{\begin{array}{l}
1 \text { for } x \geq 1 \\
0 \text { for } x<1
\end{array}\right.
$$


Neurons in this layer correspond to binary hyperplane decision rules. The weights connecting these two layers correspond to the way of occurrence of hyperplane attributes in rules. For instance, let the $k$-th minimal decision rule $\tau_{k}$ be of the form

$$
\left(H_{2}(u)<0\right) \&\left(H_{4}(u) \geq 0\right) \&\left(H_{7}(u)<0\right) \Rightarrow d(u)=v_{4}
$$

Then the corresponding weights leading to $k$-th neuron in second hidden layer take the following values:

$$
w_{j 5}=\left\{\begin{array}{c}
\frac{1}{3} \text { for } j=4 \\
-\frac{1}{3} \text { for } j=2 \text { or } 7 \\
0 \text { otherwise }
\end{array}\right.
$$

Thus, according to the above example, the $k$-th neuron in the second hidden layer will be active (its threshold function will reach 1) for some $u \in U$ iff $u$ satisfies conditions of the decision rule 2 .

For every decision value we construct one neuron in output layer, which results in $n_{d}$ outputs from the network. The $l$-th output is supposed to be active iff given object put into the network belongs to corresponding decision class $C_{l}$. To achieve such a behavior we link every decision rule neuron only with the output neuron corresponding to decision value indicated by decision rule. Thus, in case of our example, the weights between $k$-th neuron in the second hidden layer and the output layer are as follows:

$$
w_{k l}=\left\{\begin{array}{c}
1 \text { for } l=4 \\
0 \text { otherwise }
\end{array}\right.
$$

All neurons in the output layer receive threshold functions

$$
\text { out }_{l}(x)=\left\{\begin{array}{l}
1 \text { for } x \geq 1 \\
0 \text { for } x<1
\end{array}\right.
$$

\subsection{Modifications of the weights}

The above neural network, although clear and valid in its construction, does not express as much as it can yet. First of all, it does not deal with non-deterministic decision rules which are often the only way to derive any information from data. Let us go back to the example of decision rule (2) and assume that it was stated with some degree of approximation not less than 0.9 , where the value

$$
P\left(d=v_{4} \mid H_{2}<0, H_{4} \geq 0, H_{7}<0\right)=0.9
$$

corresponds to frequency of occurrence of $v_{4}$ as a decision value for subspace $C_{4}^{L, H_{2}} \cap C_{4}^{U, H_{4}} \cap C_{4}^{L, H_{7}}$ corresponding to conditions of decision rule. In this case we propose to replace previous output functions by out $(x)=x$ and link output neurons with weights $w_{k l}$ corresponding to frequency of decision value $v_{l}$ conditioned by decision rule $\tau_{k}$. Then, answering with decision value with the highest value of output function, we obtain the same classification as in 
case of decision rules. Additional information about degrees of approximation for applied rules can be derived as well.

One should realize that in case of non-deterministic rules frequencies of decision values may be often similar under given conditions. In fact, to evaluate degrees of approximation for non-deterministic decision rules we need a measure not corresponding with concrete decision values, like e.g.

$$
Q\left(\tau_{k}\right)=\sum_{v_{l} \in V_{d}}\left(P\left(d=v_{l} \mid \tau_{k}\right)\right)^{q}
$$

where $q>1$. Now, one can express the meaning of particular hyperplanes with respect to given decision rule by computing the change of $Q$ caused by rejecting particular hyperplane conditions. Let us denote by $\rho_{k j}$ decision rule $\tau_{k}$ without the $j$-th component $\tau_{k j}$. Then, for any $j=1, . ., n_{h}$ and $k=1, . ., n_{r}$ we would like to put

$$
w_{j k}= \pm \frac{1}{N_{k}} \cdot\left(Q\left(\tau_{k}\right)-Q\left(\rho_{k j}\right)\right)
$$

Remark. If one regards function (4) as the degree of approximation of decision classes, then the factor $1 / N_{k}$ is due to normalize weights coming into the neuron corresponding to the $k$-th decision rule. Each decision rule is minimal in sense that $Q$ may only decrease after rejecting any hyperplane condition. Thus, the sign \pm is adjusted just for denoting the position of points in $\tau_{k}$ with respect to the $j$-th hyperplane (compare with (3)).

\subsection{Interpretation of neuron functions}

To improve flexibility of learning, replacing original threshold functions with continuous ones should be performed. In fact, such a change enables to encode more information within our network model. Let us consider the class of (rescaled) sigmoidal functions of the form

$$
h_{j}(x)=\frac{2}{1+e^{-\alpha_{j} x}}-1
$$

for hyperplane layer. Parameters $\alpha_{j}$ express degrees of vagueness for particular hyperplanes. Parallel nature of computations along the neural network justifies searching for such parameters locally for each $H_{j}$ with respect to other hyperplanes, by applying adequate statistical or entropy-based methods (compare with $[3],[10])$.

Degrees of vagueness, proportional to the risk of basing on corresponding hyperplane cuts, find very simple interpretation. Let us weaken decision rule thresholds by replacing initial function $r_{k}$ by

$$
r_{k}(x)=\left\{\begin{array}{l}
1 \text { for } x \geq 1-\varepsilon_{k} \\
0 \text { for } x<1-\varepsilon_{k}
\end{array}\right.
$$

where parameter $\varepsilon_{k}$ expresses the degree of belief in decision rule supported by $\tau_{k}$ or, more precisely, in the quality of hyperplanes which generate it. Then, 
for fixed $\varepsilon_{k}$, increasing $\alpha_{j}$ for some $H_{j}$ occurring in $\tau_{k}$ implies that for objects which are "uncertain" with respect to the $j$-th cut function $r_{k}$ equals to 0 and no classification is obtained.

If one wants to modify functions in the second hidden layer similarly as in the first, the idea of extracting initial weights from the degrees of precision for reasoning with given hyperplanes as conditions should be followed. We claim that formulas for the decision rule functions should be derived from the shapes of functions in the previous layer. Thus, for function

$$
r_{k}(x)=\frac{1}{1+e^{-\beta_{k} x}}
$$

corresponding to decision rule $\tau_{k}$, the quantity of $\beta_{k}$ is given by formula

$$
\beta_{k}=\sum_{j=1}^{h} \alpha_{j} \cdot\left|w_{j k}\right|
$$

\section{Applications to tuning of conditional hyperplanes}

Modifications introduced for initial model of hyperplane-based neural network enable to include necessary information for improvement of decision classification. Obviously, described changes may cause that our network become not consistent with decision rules for some part of training objects. It means that, e.g. for majority frequency rules, the output corresponding to a decision value pointed by some rule may not be the one with the highest value of the output function. Such inconsistency, however, is justified by computing all weights and neuron functions from decision table itself. Moreover, we have still possibility of tuning the network by the wide range of learning techniques.

In classical backpropagation networks ([1],[2]) update of weights is based on gradient descent technique. The backpropagation method allows us to perform learning by minimizing any differentiable error function. The update for any weight $w$ in the network is given by:

$$
\Delta w=-\eta \frac{\partial \delta}{\partial w}
$$

where $\eta$ is a learning coefficient. To keep relationship with the way of computing initial weights in the learning process, we consider error functions or the form

$$
\delta(u)=\frac{1}{q} \sum_{1 \leq l \leq n_{d}}\left(\text { out }_{l}(u)-i n_{l}(u)\right)^{q}
$$

where

$$
\operatorname{out}_{l}(u)=\sum_{1 \leq k \leq n_{r}} \frac{w_{k l}}{1+\exp \left(-\beta_{k} \sum_{1 \leq j \leq n_{h}} w_{j k}\left(\frac{2}{1+\exp \left(-\alpha_{j} H_{j}(u)\right)}-1\right)\right)}
$$

and

$$
i n_{l}(u)=\left\{\begin{array}{c}
1 \text { for } d(u)=v_{l} \\
0 \text { otherwise }
\end{array}\right.
$$


We can also use the cumulative error given by

$$
\delta(U)=\frac{1}{q \cdot \operatorname{card}(U)} \sum_{u \in U} \sum_{1 \leq l \leq n_{d}}\left(o u t_{l}(u)-i n_{l}(u)\right)^{q}
$$

In this case we back-propagate the global error from the whole set of objects.

Once more we would like to stress that error functions given above correspond to the quality measure $Q$ introduced before. Thus, if one would like to consider hyperplane decision rules minimal in sense of another criterion, the way of measuring classification error should be verified properly.

In classical neural network learning we may manipulate with some coefficients to control the learning process ([1],[2]). In our approach we may use this ability in order to introduce some meaning for such operations. Change of weights in first hidden layer corresponds to the change of elevation of hyperplanes. Hence, by setting constraints for value of learning coefficients we may induce the learning in case we e.g. do not want the hyperplanes to change too rapidly. The standard tricks from the network learning, like momentum factor ([2]), can also be used, although they do not have explicit interpretation in terms of hyperplanes and decision trees.

During the learning process we should still remember about the interpretation of weights and functions. Starting from initial structure obtained from data by sequential algorithm for finding hyperplanes, we begin to modify weights due to given learning method. Then, however, for possibly improved classification we cannot determine how the decision rules behave over data actually. Another point is to keep decision rules minimal for foregoing hyperplane weights to make the whole process more clear. Thus, it turns out to be very important to preserve the balance between what is derived from the learning process and what is obtained from described construction.

\section{Conclusions}

In our paper we discussed the connection between rough set techniques for realvalued decision tables and analogous methods based on neural networks. After introducing initial framework, we described basic model of neural network corresponding to hyperplane-based methods developed in [5],[6]. Clear interpretation of its structure with respect to weights and neuron functions enabled us to join computational power of neural networks with simplicity of rough set decision techniques.

Applications of obtained framework e.g. to tuning hyperplane decision rules need further work on implementation methods. The most serious problem is how to keep the balance between convergence of the tuning process and simplicity of its outcome interpretation. Optimization of such factors like decision and condition scaling or, e.g., safety of decision rules requires methods of parallel learning on different levels. Thus, from practical point of view, one has to specify the balance between the amount of information wanted and effectiveness of application to particular data. 
Presented methods should be compared also with other approaches. For instance, one can consider the problem of handling continuous decision. Then our neural network model becomes strongly related to fuzzy inference ([4],[9]). It can be treated as one of regression problems, well known in machine learning, statistics, neural network and fuzzy sets.

\section{References}

1. Hecht-Nielsen, R. (1990). Neurocomputing. Addison-Wesley, New York.

2. Karayiannis, N.B., Yenetsanopoulos, A.N. (1993). Artificial Neural Networks : Learning Algorithms, Performance Evaluation and Applications. Kluwer, Dortrecht.

3. Kohavi, R., Sahami, M. (1996). Error-Based and Entropy-Based Discretization of Continuous Features. In Simoudis E., Han J. \& Fayyad U.M.(eds.) Proc. of the Second International Conference on Knowledge Discovery \& Data Mining. August 1996, Portland, Oregon, pp. 114-119.

4. Kruse, R., Gebhardt, J., Klawonn F. (1994). Foundations of Fuzzy Systems. Wiley, Chichester.

5. Nguyen, H.S., Nguyen, S.H. (1996). From Optimal Hyperplanes to Optimal Decision Tree. In: Proc. of the IV International Workshop on Rough Sets, Fuzzy Sets and Machine Discovery RSFD'96. Nov.6-8, 1996, Tokyo, Japan, pp.82-88.

6. Nguyen, H.S., Nguyen, S.H., Skowron, A. (1996). Searching for Features defined by Hyperplanes. In: Z.W. Raś, M. Michalewicz (eds.), Proc. of the IX International Symposium on Methodologies for Information Systems ISMIS'96. June 1996, Zakopane, Poland. Lecture Notes in AI 1079, Berlin, Springer Verlag, pp.366-375.

7. Nguyen, H.S., Skowron, A. (1995). Quantization of Real-valued Attributes, Rough Set and Boolean Reasoning Approaches. In: Proc. of the Second Joint Annual Conference on Information Sciences. Wrightsville Beach, North Carolina, Sept 28 Oct 1, 1995, USA, pp.34-37.

8. Skowron, A., Rauszer, C. (1992), The Discernibility Matrices and Functions in Information Systems. In: R.Slowinski (ed.), Intelligent Decision Support - Handbook of Applications and Advances of the Rough Sets Theory. Kluwer, Dordrecht, pp.331-362.

9. Szczuka, M., Ślęzak, D. (1997). Hyperplane-based Neural Networks for Real-valued Decision Tables. Proc. of the RSSC'97. Raleigh, NC, USA. pp. 265-268.

10. Vapnik, V.N. (1995). The Nature of Statistical Learning Theory. Springer-Verlag, New York. 